\title{
Creación Colaborativa de Recursos Educativos Abiertos: Experiencias de la Iniciativa LATIn
}

\author{
Manuel Podetti ${ }^{1}$, Virginia Rodés ${ }^{1}$, Xavier Ochoa $^{2}$, Ismar Frango $^{3}$, Alén Pérez \\ Casas $^{1}$ \\ ${ }^{1}$ Programa de Entornos Virtuales de Aprendizaje, Comisión Sectorial de Enseñanza \\ Universidad de la República (UdelaR) \\ J.E. Rodó 1854 - Montevideo - Uruguay \\ ${ }^{2}$ Escuela Superior Politécnica del Litoral (ESPOL) Campus Gustavo Galindo - CTI, \\ Km. 30.5 Vía Perimetral, Guayaquil - Ecuador \\ ${ }^{3}$ Universidade Presbiteriana Mackenzie (UPM) R. da Consolação 930, 01302-907, São \\ Paulo, SP - Brazil
}

\begin{abstract}
Summary. In this paper the processes performed by the Latin Project to create the Latin American Open Textbooks Initiative are presented. To this end, a collaborative design methodology, appropriate technological platforms and support a strategy for the generation and adoption of open text books in the partner universities of the project were implemented. The project objective was to increase the accessibility of low-income students to textbooks and generate content from and for Latin America, thinking in different contexts, cultures and languages.
\end{abstract}

Resumen. En este trabajo se presentan los procesos realizados por el Proyecto LATIn para crear la Iniciativa Latinoamericana de Libros de Texto Abiertos. Para tal fin se diseño una metodología colaborativa, se implementaron plataformas tecnológicas adecuadas y se apoyo en una estrategia para la generación y adopción de libros de textos abiertos en las Universidades socias del proyecto. El objetivo del proyecto fue aumentar la accesibilidad de estudiantes de bajos recursos a libros de texto y generar contenidos desde y para Latinoamérica, pensando en sus diferentes contextos, culturas e idiomas.

Palabras Clave: Libros de Texto Abiertos, Recursos Educativos Abiertos (REA), Libros digitales, TIC, Enseñanza, Creative Commons. 
CBIE-LACLO 2015

Anais dos Workshops do IV Congresso Brasileiro de Informática na Educação (CBIE 2015)

\section{Introducción}

El Proyecto LATIn (Latinoamerican Open Textbook Books Initiative), conformado ${ }^{1}$ por nueve universidades de América Latina y tres europeas, inicia a principios del 2012, con el apoyo de la Unión Europea en el marco de su Programa ALFA III ${ }^{2}$. Sus principales objetivos se orientan a disminuir el problema del alto costo de los libros de texto en la Educación Superior de América Latina y a generar contenidos educativos desde y para Latinoamérica, tomando en cuenta sus diferentes contextos, culturas e idiomas. Para ello se propone crear la Iniciativa Latinoamericana de Libros de Texto Abiertos.

Si bien los estudiantes latinoamericanos tienen acceso a más de 60 Universidades públicas, existe un gasto no siempre visible que les impide llevar de modo adecuado sus cursos. Se trata de los altos costos de los libros de texto. Un estudio reciente revela que un estudiante de la Universidad de São Paulo, el mayor instituto de enseñanza superior de carácter público de Brasil, tiene un costo promedio anual de 1,900€ en compra de libros de texto. Esto equivale al $67 \%$ del salario mínimo brasileño (2.820€ al año) [Craveiro, Machado y Ortellado 2008]. Esta situación empeora en otros países Latinoamericanos.

Una de las raíces de estos altos costos es que la mayoría de los libros de texto se producen fuera de la región, problemática que no está relacionada con la falta de capacidad de producción, sino con la dificultad que tienen los docentes o autores locales para publicar y distribuir sus libros. En consecuencia, la mayoría de los libros de texto no están traducidos y/o adaptados al contexto de la Educación Superior en Latinoamérica.

Por estos motivos es que la principal acción del proyecto LATIn radica en la creación y diseminación de la Iniciativa Latinoamericana de Libros de Texto Abiertos a nivel universitario.

En el presente artículo se presentan los principales componentes del Proyecto LATIn. En la sección 2 se resume la investigación realizada por el Proyecto LATIn entorno al estado del arte en cuanto a iniciativas de libros de texto abiertos a nivel mundial y metodologías de escritura colaborativa para libros de texto abiertos. En la sección 3 se muestra un resumen del análisis realizado sobre las percepciones, actitudes y prácticas de los estudiantes en relación a los libros de texto en sus diferentes formatos, realizada entre las Universidades del consorcio LATIn; mientras que en la sección 4 se desarrolla

\footnotetext{
${ }^{1}$ Escuela Superior Politécnica del Litoral, Ecuador (ESPOL); Universidad Autónoma de Aguascalientes (UAA), Universidad Católica de San Pablo, Perú (UCSP); Universidade Presbiteriana Mackenzie, Brasil(UPM); Universidad de la República, Uruguay (UdelaR); Universidad Nacional de Rosario, Argentina(UR); Universidad Central de Venezuela, Venezuela (UCV), Universidad Austral de Chile, Chile (UACH), Universidad del Cauca, Colombia (UNICAUCA), Katholieke Universiteit Leuven, Bélgica (KUL), Universidad de Alcalá, España (UAH), Université Paul Sabatier, Francia (UPS)

${ }^{2}$ Proyecto ALFA III EuropeAid/130999/C/ACT
} 
el proceso de implementación de la Iniciativa Latinoamericana de Libros de Texto Abiertos (LATIn). Finalmente, la sección 5 presenta las principales acciones encaminadas para favorecer la diseminación de la misma en América Latina.

\section{Estado de Arte}

\subsection{Experiencias de iniciativas de libros de texto abiertos}

La primera acción del proyecto fue investigar el estado del arte sobre experiencias de libros de texto abiertos a nivel mundial. Para ello se puso foco en identificar y describir los procesos de implementación y adopción de cada iniciativa. Si bien la mayoría de las experiencias se encontraron en Europa y EE.UU., se registraron algunas experiencias incipientes en Latinoamérica y Asia.

Como se detalla en [1], desde hace una década han comenzado a surgir iniciativas que tienen como finalidad reducir los costos de los libros de texto en diferentes ámbitos académicos y de esta forma, lograr un libre acceso a los contenidos educativos. Entre las identificadas se puede mencionar a MERLOT [http://taste.merlot.org/opentextbooks.html], que almacena una gran colección de libros abiertos. Así también AIM [http://www.aimath.org/textbooks/], la cual busca fomentar la creación de libros de texto abierto en el área de Matemáticas, y Connexions [http://cnx.org/] en el cual se puede almacenar y compartir materiales educativos creados en base a pequeñas unidades de contenido llamados módulos, los cuales pueden ser organizados como cursos, libros, reportes, etc. Health Education Assets Library (HEAL) [http://www.healcentral.org/], por su parte, almacena materiales digitales para la educación en ciencias de la salud entre otras iniciativas.

En el contexto latinoamericano se identifican algunos casos incipientes de creación colaborativa de textos. Uno de ellos es el caso Hipernexus en la Universidad Pontificia Bolivariana, proyecto dirigido a profesores y estudiantes y desarrollado por un grupo de investigación sobre la educación en entornos virtuales. Otro caso estudiado fue la Biblioteca Virtual de Ciencias Sociales de América Latina y el Caribe, CLACSO [ http://www.clacso.org.ar/ ]. Ambos casos dan cuenta de experiencias piloto en la creación y uso de textos, implementados en una etapa temprana.

El principal resultado obtenido del análisis del estado del arte realizado fue la posibilidad de extraer un conjunto de características o dimensiones, que se muestran intrínsecamente relacionadas con las estrategias que deben aplicarse para la adopción de una iniciativa de libros de texto abiertos: dimensión temporal (fases de la adopción), dimensión de procesos (diseminación, promoción, formación) y dimensión social (actores y contextos). El éxito en el proceso de adopción está fuertemente ligado a la adecuada selección de las instancias que se asignarán a estas dimensiones. En ese sentido, con base en la revisión de las estrategias estudiadas, se diseñó la Estrategia de Adopción del Proyecto LATIn particularizada para el contexto latinoamericano.

\subsection{Metodologías de escritura colaborativa para libros de texto abiertos}


Paralelamente se realizó un estudio de estado del arte de las metodologías dedicadas a la escritura colaborativa de contenidos abiertos [2] En dicho trabajo se analizaron esas metodologías a partir de la noción general de colaboración para luego profundizar en metodologías específicas para la creación de libros de texto de modo colaborativo.

La creación colaborativa de libros de texto abiertos se ubica en el ámbito de estudio denominado Computer Supported Cooperative Work (CSCW) [3] y más específicamente de las herramientas de Groupware de escritura colaborativa. Después del análisis de la literatura se concluye que la escritura colaborativa, aunque es un fenómeno suficientemente comprendido, aún es un proceso que es re - descubierto por cada grupo involucrado en la tarea. Existen algunas pautas, como las presentadas por [4] y [5], pero su carácter general hace que sean más útiles para analizar un proceso de colaboración ya existente que para guiar el desarrollo de una estrategia metodológica. El advenimiento de nuevas maneras de comunicarse e interactuar, generados por el surgimiento de las tecnologías de la Web 2.0, vuelven a abrir la discusión sobre la forma de organizar la escritura colaborativa en grupos. Se puede concluir que el rango de las estrategias para la escritura colaborativa de libros de texto abiertos varían de acuerdo a las necesidades y el contexto. No existe un tipo de metodología que pueda ser útil para cada caso y situación. Por esa razón, la propuesta de metodología para LATIn requirió que fuese adaptable a los diferentes modelos de funcionamiento de cada grupo y que permitiera incorporar tipos de colaboración derivados de las nuevas tecnologías de Internet.

\section{Relación de los estudiantes con los libros de texto en sus diferentes formatos}

Con el objetivo de obtener una caracterización de los niveles de acceso a los libros de texto en sus diversos formatos por parte de estudiantes de universidades de América Latina y orientar la toma de decisiones para el desarrollo de la plataforma colaborativa de libros de texto abiertos del proyecto, se realizó un estudio de Análisis de Necesidades $[6]$.

El estudio se desarrolló en base a una consulta abierta en línea aplicada a estudiantes de las Universidades Latinoamericanas que forman parte del Proyecto LATIN. Se orientó a obtener perfiles de acceso de los estudiantes a los libros de texto necesarios para sus carreras, evaluar la predisposición hacia el uso de libros en formatos digitales y abiertos e identificar las características deseables que los libros de texto digitales deben tener desde la perspectiva del estudiante como usuario.

Del estudio realizado se obtuvo la respuesta proveniente de 2058 individuos provenientes de 5 universidades: ESPOL, UACH, UDELAR, UCSP y UNR ${ }^{3}$.

\footnotetext{
${ }^{3}$ Escuela Superior Politécnica del Litoral, Universidad Austral de Chile, Universidad de la República, Universidad Católica de San Pablo, Universidad Nacional de Rosario.
} 


\section{CBIE-LACLO 2015}

Anais dos Workshops do IV Congresso Brasileiro de Informática na Educação (CBIE 2015)

Si bien la muestra obtenida no es estadísticamente representativa, en la medida en que es amplia y participativa, permite elaborar perfiles generales y compararlos entre universidades y/o tipologías de usuarios.

Considerando que los estudiantes son los destinatarios finales del sistema y de los contenidos desarrollados, se plantearon algunas preguntas disparadoras del cuestionario de la consulta en línea. Entre ellas se destacan:

Se buscó asimismo que la consulta fuera similar a otros estudios realizados en iniciativas similares fuera de la región [7], [8], [9], con el fin de poder realizar estudios comparados. A continuación presentamos los principales hallazgos obtenidos.

\subsection{Acceso a los libros de texto}

Respecto del acceso, algo más del $40 \%$ de los estudiantes declaran que no pueden acceder a textos obligatorios, entre ellos, un tercio declaran que no tienen acceso a algunos de los textos fundamentales.

\subsection{Impacto de la compra de libros de texto en el presupuesto}

En cuanto al impacto de la compra de libros de texto en el presupuesto de los estudiantes es claro que existe una problemática al respecto. Un $50 \%$ de los encuestados afirma que la compra de libros es al menos una parte significativa de su presupuesto y un $24 \%$ directamente no compra libros de texto. Dejando sólo un $26 \%$ de estudiantes encuestados que no tendría obstáculos económicos para comprar libros de texto universitarios.

Respecto de los tipos de texto que utilizaron durante este semestre en el estudio realizado se encontró que la mayoría de los estudiantes utilizan una serie diversa de recursos, tanto en papel como digitales. El formato más utilizado es la "Fotocopia en papel de capítulos sueltos de libros de texto" que es usado por el 63\% de los estudiantes.

Los "Libros enteros fotocopiados en papel" le siguen de cerca con una proporción similar. Las bibliotecas son la alternativa para el acceder a los libros para el 54\% de los estudiantes. Una proporción muy similar (48\%) es la que declara que utilizaron "libros digitales bajados de la web, sin las licencias correspondientes".

Un dato un tanto inesperado es la relativamente alta (36\%) proporción de los que optaron por los "libros o textos digitales abiertos, con licencias Creative Commons u open source", frente al 5\% que utilizó libros comprados por internet y esta respuesta recibe proporciones similares en las diversas universidades. 


\section{CBIE-LACLO 2015}

Anais dos Workshops do IV Congresso Brasileiro de Informática na Educação (CBIE 2015)

Solo un 7\% declara que "no suele acceder a libros de texto en formato digital", pero la gran mayoría (58\%) opta por "imprimirlos en papel para leerlos", frente al $27 \%$ que los lee en la pantalla de PC y el 3\% que lo lee en otros dispositivos, como tablet, móviles o lectores de libros electrónicos.

Estos datos indican una buena penetración de los textos en formatos digitales y una explícita declaración de interés en los recursos abiertos. Sin embargo, la preferencia por el papel como soporte para la lectura sigue siendo muy importante, entre otros motivos por la relativamente escasa difusión de los dispositivos portátiles específicos para la lectura de libros, 3,4\% poseen lector de ebook 4,4\% disponen de Tablet. El dispositivo más mencionado es la computadora portátil tipo Notebook (58\%) y Netbooks (17\%) frente al PC de Escritorio con un 52\%. Hay que considerar que hay un $11 \%$ que no disponen de ninguno de estos dispositivos (PC de Escritorio, Notebook, Netbook, Tablet o Lector de Ebook) y que la situación más común (el 60\%) poseen sólo uno de estos dispositivos y que un $25 \%$ poseen dos.

En el capítulo donde se indaga específicamente sobre las características de los libros de texto digitales, se encontró que la disponibilidad es la más apreciada. El 92\% valoró como "muy importante" que los libros de texto universitarios estén "disponibles en internet", y casi ninguno consideró esta una característica "nada importante". Esta característica, la necesidad de tener los libros disponibles en internet, se repite en el estudio realizado en [10], donde, la disponibilidad online es la principal característica elegida por el $98 \%$ de los encuestados.

\section{Implementación de la iniciativa}

Apoyados en los estudios realizados el Proyecto LATIn confirmo la necesidad de generar cuatro plataformas que den soporte a la Iniciativa Latinoamericana de Libros de Texto Abiertos: una plataforma social, una de escritura, otra de publicación y una editorial.

\subsection{La plataforma social}

Cuyo objetivo es brindar un entorno donde los docentes se encuentren y compartan sus conocimientos y experiencias a nivel académico y profesional. La plataforma basada en software libre (ELGG) permite que cada docente cree una usuario con su respectivo perfil y pueda generar y/o participar en comunidades temáticas donde dialogar e intercambiar información. Si bien no es una condición excluyente, el objetivo es que de esas comunidades puedan surgir libros de texto abiertos realizados colaborativamente. Desde cada una de las comunidades se pueden crear grupos de escritura en donde pueden participar todos o parte de los participantes de esa comunidad. Al crear un grupo 
de escritura automáticamente se genera un acceso directo al template del libro en la plataforma de escritura, desde allí pueden comenzar a escribir el texto. http://comunidad.proyectolatin.org/

4.2 Plataforma de escritura colaborativa, esta basada en Etherpad, editor web que permite la colaboración en tiempo real y Booktype enfocado en autoría, edición y publicación de textos en diferentes formatos como PDF, ePub, ODT, mobi y HTML. http://escritura.proyectolatin.org/.

4.3 Una plataforma de publicación, desde donde se publican los libros de texto del Proyecto LATIn, licenciados con una licencia Creative Commons: ReconocimientoCompartirIgual 3.0 Unported (CC BY-SA 3.0) El mismo se eligió debido a que permite a estudiantes y profesores, leer, imprimir, descargar, compartir, adaptar, traducir y modificar los libros de texto.

4.4 La Editorial Abierta, en la que se publicarán libros de LATIn, así como otros que surjan de acuerdos con editoriales universitarias y privadas. En este sentido los libros publicados no se limitarán a la licencia 3.0 de Creative Commons, como es el caso de los libros publicados por LATIn. La editorial contará con una tienda virtual y se apoyará en una red de impresoras a demanda. Si bien el Proyecto LATIn finaliza en 2014, toda las plataformas quedarán operativas y funcionando, incluso se mejorarán. En esta línea la Editorial Abierta cumplirá una función crucial a la hora de dotar de continuidad y sustentabilidad a la Iniciativa Latinoamericana de Libro de Texto Abiertos.

\section{Estrategias de Adopción:}

Paralelamente a la generación de las plataformas se llevan adelante diversas acciones vinculadas a una Estrategia de Adopción, organizadas en tres áreas: difusión, promoción y formación

\subsection{Difusión}

Para realizar la difusión se utilizarón los medios tradicionales (prensa escrita, radio y televisión), no tradicionales (blogs y redes sociales) así como presenciales (charlas y conferencias). Para potenciar la institucionalidad se eligieron conceptos o ideas fuerza a las que debería asociarse LATIn, como Libros de textos abiertos, recursos educativos abiertos (REA), Creative Commons, creación colaborativa y educación abierta.

Una de las principales acciones de comunicación fue la Convocatoria a la presentación de propuestas para la publicación de Libros de Textos Abiertos Universitarios para América Latina, en el marco del cual se se ofreció apoyo económico a los docentes que elaboren los primeros libros de texto abiertos de la iniciativa.

Gracias a la difusión generada por esta convocatoria, ingresaron a la Plataforma Social del proyecto 5.100 visitantes únicos, lo cuales generaron 8.897 visitas entre el 15 de mayo y el 10 de octubre de 2013, según las analíticas de Google. Esto permitió conectar 


\section{CBIE-LACLO 2015}

Anais dos Workshops do IV Congresso Brasileiro de Informática na Educação (CBIE 2015)

a más de 500 docentes e investigadores de toda Latinoamérica, quienes generaron más de 100 comunidades sobre diversas temáticas, desde donde se presentaron más de 60 propuestas de libros de texto abiertos. De éstas fueron seleccionadas 25 , que involucran a 190 docentes de diferentes universidades de Latinoamérica, ya que la consigna más innovadora del proyecto fue que los libros deben realizarse colaborativamente.

Luego de realizada la convocatoria se continuó difundiendo el proyecto a través de diferentes canales: correo electrónico y portales y medios de las universidades socias.

En cuanto a los medios sociales, se decidió generar un perfil en Twitter y Linkedin y una página de Facebook, desde donde se difunden acciones institucionales del proyecto e información relacionada a las ideas fuerza. Para ellos se "sigue" a usuarios o instituciones referentes en las temáticas y se "retwitea" o "republica" sus contenidos en los perfiles de LATIn. Esto permite un triple objetivo de comunicación, difundir conceptos como la educación abierta entre las personas e instituciones que "siguen" a LATIn, generar una asociación entre LATIn y referentes en la temática y lograr que estas mismas instituciones difundan el Proyecto LATIn entre sus contactos y redes, potenciando nuevamente los objetivos, asociación a referentes y difusión de la iniciativa.

5.2 Promoción: A diferencia de la difusión, la promoción se orienta a que se tomen las acciones necesarias para que se adopte la Iniciativa en las diferentes universidades. Para ello se lograron encuentros con referentes de las área para promover y fomentar la adopción, autoría y publicación de libros de texto abierto, así como las medidas complementarias que facilitan este proceso en las diferentes áreas encargadas de los aspectos legales, educativos, operativos y tecnológicos.

\subsection{Formación:}

Se refiere a las acciones que se realizan en las instituciones para capacitar y formar a los actores, favoreciendo la apropiación de las estrategias educativas necesarias para hacer frente a la creación, uso y reutilización de libros de texto abiertos y colaborativos. En particular se han puesto en línea dos cursos abiertos: Introducción a la Educación Abierta (https://canvas.instructure.com/courses/813222), curso adaptado del diseñado por David Wiley (Universidad de Utah, EE.UU.) precursor y referente indiscutido en el tema de los Recursos Educativos Abiertos y la Educación Abierta y Libros de Texto Abiertos (https://canvas.instructure.com/courses/813223).

\section{Conclusiones}

El Proyecto LATIN finalizó en el 2014, su informe final puede verse en [11], así como consultar los primeros 23 libros generados por la iniciativa, que ya han sido utilizados por alumnos de las universidades socias [12] 


\section{CBIE-LACLO 2015}

Anais dos Workshops do IV Congresso Brasileiro de Informática na Educação (CBIE 2015)

Actualmente las plataformas (social, escritura y publicación) se encuentran en funcionamiento y siendo utilizadas por las universidades participantes, mientras que han mostrado interés otras universidades de la región.

Las principales dificultades del proyecto radicaron en su carácter innovador, lo que generó en el propio equipo problemas a la hora de resolver los desafîos de implementar las herramientas de escritura colaborativa, o diseñar las estrategias de adopción que involucraban cambios culturales profundos en lo que respecta a las prácticas de licenciamiento y autoría. Del mismo modo la elaboración de los libros de manera colaborativa, implicó para los docentes un gran desafío. Si bien lograron culminar los textos, fue clara en muchos casos la falta de formación en recursos educativos abiertos, licencias libres y sobre todo adaptarse a compartir y crear de modo colaborativo.

Creemos que esta experiencia constituye un antecedente relevante e innovador de modelos de creación colaborativa de recursos educativos abiertos, en una región en la cual se requiere muchísimas más iniciativas y desarrollo en la temática. Asimismo da cuenta de un modelo alternativo de creación de textos para el ámbito universitario, en donde la inversión se dirige a ofrecer incentivos a los docentes en contraposición al pago a editoriales.

\section{Referencias}

1. Rodés, V. Solano, C. Jorge. Maturana, J. Hernandez, Y. Cuadros, E. Podetti M. (2012) "Strategies for Implementing the Adoption of Open Textbooks Initiatives: State of the Art" Review. International Journal of e-Education, e-Business, e- Management and eLearning, v.: 3 1, 2013.Singapore ; ISSN: 20103654 ; DOI: 10.7763/IJEEEE.2013.V3.192.

2. Casali , A., Silva A., Alves, C., Deco, Claudia, Frango I., Et Al. (2012) "Collaborative Methodologies for Writing Open Educational Textbooks: a State-of art Review" Proceedings del I Workshop Recursos Educacionais Abertos: Questões para globalização e localização y II International Symposium on OER: Issues for globalization and localization, CBIE; Río de Janeiro.

3. Baecker, R. M., Grudin, J., Buxton, W. A. S., Greenberg, S. (1995) "Readings in HumanComputer Interaction: Towards the Year 2000" (Second Edition) Morgan Kaufmann Publishers, Inc.

4. Ede, L. and Lunsford, A.: Singular Texts/Plural Authors (1990) "Perspectives on Collaborative Writing”. Southern Illinois University Press. 


\section{CBIE-LACLO 2015}

Anais dos Workshops do IV Congresso Brasileiro de Informática na Educação (CBIE 2015)

5. Posner, I. \& Baecker, R. (1993) "How people write together". Proceedings of the International Conference on System Sciences, 25, 127-137.

6. Rodés, V., Ochoa, X., Silveira, I. F., Casas, A. P. (2012) "Percepciones, actitudes y prácticas respecto a los libros de texto, digitales y en formatos abiertos por parte de estudiantes de universidades de América Latina". In: II Workshop em Recursos Educacionais Abertos, 2012, Rio de Janeiro. Anais do CBIE 2012 - Workshops.

7. Florida Distance Learning Consortium. Florida Student Textbook Survey. Tallahassee, 2011.

8. McKiel., A. 2008 Global Student E-book Survey. Ebrary. United Kingdom 2008

9. McKiel. A 2012 Global Student E-book Survey. Ebrary. United Kingdom 2012.

10. Rodés, V,.; Pérez, A.; Ochoa, X.; Frango , I. 2012. Percepciones, actitudes y prácticas respecto a los libros de texto, digitales y en formatos abiertos por parte de estudiantes de universidades de América Latina. I Workshop Recursos Educacionais Abertos: Questões para globalização e localização II International Symposium on OER: Issues for globalization and localization . 28 e 29 de Novembro de 2012, Brasil. Anais dos Workshops do Congresso Brasileiro de Informática na Educação. ISSN: 2316-8889. Disponible en: http://www.br-ie.org/pub/index.php/wcbie/article/view/1893

11. LATIn, 2015. Iniciativa Latinoamericana de Libros de Texto Abiertos. Documento final de la Iniciativa de Libros de Texto Abiertos LATIn. Disponible en: http://latinproject.org/pdfs/final_document.pdf

12. Frango, I.; Silva, A.; Rodés, V.; Valdivia, Y. 2015 "Análise do uso de livros-texto digitais abertos no contexto da Educação Superior na América Latina”. Revista Novas Tecnologias na Educação (RENOTE) v. 13, n. 1 (2015). 